PROCEEDINGS OF THE

AMERICAN MATHEMATICAL SOCIETY

Volume 137, Number 7, July 2009, Pages 2265-2271

S 0002-9939(09)09807-4

Article electronically published on January 30, 2009

\title{
RINGS WHOSE MODULES ARE DIRECT SUMS OF EXTENDING MODULES
}

\author{
NOYAN ER \\ (Communicated by Birge Huisgen-Zimmermann)
}

\begin{abstract}
We prove that for a ring $R$, the following are equivalent: (i) Every right $R$-module is a direct sum of extending modules, and (ii) $R$ has finite type and right colocal type (i.e., every indecomposable right $R$-module has simple socle). Thus, in this case, $R$ is two-sided Artinian and right serial, and every right $R$-module is a direct sum of finitely generated uniform modules. This property of a ring is not left-right symmetric. A consequence is the following: $R$ is Artinian serial if and only if every $R$-module is a direct sum of extending modules if and only if $R$ is left serial with every right $R$-module a direct sum of extending modules.
\end{abstract}

\section{INTRODUCTION}

$R$ will denote an associative ring with identity and modules will be unital. A module $M$ is called extending if every submodule of $M$ is essential in a direct summand of $M$. Extending modules generalize (quasi-)injective, semisimple, and uniform modules and have been extensively studied over the last few decades (see [3] for a detailed account of such modules). We will consider the following properties for $R$ :

$(*)_{r}\left(\right.$ resp. $\left.(*)_{l}\right)$ : Every right (resp. left) $R$-module is a direct sum of extending modules.

$(*)$ : Both $(*)_{r}$ and $(*)_{l}$ hold.

An Artinian ring $R$ is said to have right colocal type if every finitely generated indecomposable right $R$-module has simple socle. Such rings and algebras have been investigated by several authors including Makino [8, Sumioka [14-[15], Tachikawa [16-[17, and a special case by Fuller [6]. Artinian serial rings clearly have finite type as well as right and left colocal type.

On the other hand, characterizations of rings have been given in terms of modules which are extending (see, for example, [2, 3], 4, [5]). In particular Dung and Smith proved that $R$ is Artinian serial with $J(R)^{2}=0$ if and only if every right $R$-module is extending [2, Theorem 11]. Since the extending property does not carry over to (finite) direct sums, as can be seen by the example $\frac{\mathbb{Z}}{p \mathbb{Z}} \oplus \frac{\mathbb{Z}}{p^{3} \mathbb{Z}}(p$ a prime), the problem of determining rings with $(*)_{r}$ becomes difficult. In this paper, we will prove that such rings are precisely the rings of finite type and right colocal

Received by the editors April 22, 2008, and, in revised form, August 21, 2008, and October 16, 2008.

2000 Mathematics Subject Classification. Primary 16D10, 16D70, 16P20; Secondary 16G60.

(C)2009 American Mathematical Society

Reverts to public domain 28 years from publication 
type, so that they are Artinian, right (but not necessarily left) serial. Consequently, Artinian serial rings are precisely those rings with $(*)_{r}$ and $(*)_{l}$.

The following concepts will be used in our proofs: Let $M$ be a module and $A, B$, $C_{i}(i \in I)$ be submodules of $M$. $A$ is called a closed submodule of $M$ if it has no proper essential extension in $M$. $A$ is said to be a complement in $M$ of $B$ if it is a maximal element in $\{X \subseteq M \mid X \cap B=0\}$. Complements in $M$ are precisely closed submodules of $M .\left\{C_{i} \mid i \in I\right\}$ is called a local summand of $M$ if $\sum_{i \in I} C_{i}=\bigoplus_{i \in I} C_{i}$ and every finite subsum is a direct summand of $M$. $M$ is called a colocal module if it is uniform with simple socle. $M$ is called q.f.d. (quotient finite dimensional) if every factor of $M$ has finite uniform (Goldie) dimension. $R$ is called right q.f.d. if $R_{R}$ is q.f.d. $R$ is called right $Q F-3$ if there exists a faithful right $R$-module that is a direct summand of every faithful right $R$-module. For all other basic definitions and results the reader may refer to [7].

\section{RESUlts}

We need the following lemmas to prove our main result. Lemma 1 is well known, but we give a proof for completeness.

Lemma 1. Let $M$ be a module and $N$ be an essential submodule of $M$. Then, for any closed submodule $K$ of $M, K \cap N$ is a closed submodule of $N$.

Proof. Since $K$ is closed in $M, K$ must be a complement of a submodule $T$ of $M$. Now it suffices to see that $K \cap N$ is a complement of $T \cap N$ in $N$. Let $B$ be a submodule of $N$ properly containing $K \cap N$. Then $(K+B) \cap T \neq 0$. So there exists some $0 \neq t=k+b \in(K+B) \cap T$, with $k \in K$ and $b \in B$. Since $N$ is essential in $M$, there exists some $r \in R$ such that $0 \neq t r=k r+b r \in T \cap N$. Then $k r \in K \cap N \subseteq B$, so that $t r$ is a nonzero element of $T \cap N \cap B$. This proves our claim, whence the conclusion follows.

Lemma 2. A right q.f.d. ring $R$ with property $(*)_{r}$ is right Noetherian.

Proof. Let $R$ be a ring with $(*)_{r}$. Assume that $R$ is not right Noetherian. Then, by [1, Theorem 1.3], there exists a family $\left\{S_{n}: n \in \mathbb{N}\right\}$ of simple modules such that no infinite subsum of $V=\bigoplus_{n \in \mathbb{N}} E\left(S_{n}\right)$ is injective ${ }^{(\sharp)}$. Then pick an element $y \in E(V)-V$ and, by Zorn's lemma, let $T$ be a maximal element among submodules of $E(V)$ containing $V$ but not containing $y$. Then $\frac{E(V)}{T}$ is colocal. Now assume $T=X \oplus Y$, and let $E(X)$ and $E(Y)$ be injective hulls in $E(V)$ of $X$ and $Y$ respectively. Then $\frac{E(V)}{T} \cong \frac{E(X)}{X} \oplus \frac{E(Y)}{Y}$. This means that one of the latter two modules, say $\frac{E(X)}{X}$, is zero; whence $X=E(X)$ is injective.

Now we claim that we can write $T=A \oplus B$, for some noninjective extending module $A$ and some injective module $B$ : By assumption $T=\bigoplus_{\gamma \in \Gamma} A_{\gamma}$, for some nonzero extending modules $A_{\gamma}$. If $\Gamma$ is finite, then our claim follows easily by the argument preceding the claim. So assume that $|\Gamma|=\infty$. Applying Krull-RemakSchmidt on the socles we can pick a simple submodule $V_{\gamma} \subseteq A_{\gamma}$ for each $\gamma \in \Gamma$, and a one-to-one map $\gamma \rightarrow n_{\gamma}$ from $\Gamma$ into $\mathbb{N}$, with $V_{\gamma} \cong S_{n_{\gamma}}$. Since each $V_{\beta}(\beta \in \Gamma)$ is contained in a finite subsum of $\bigoplus_{n \in \mathbb{N}} E\left(S_{n}\right)$, the latter then contains an injective hull of $V_{\beta}$, which is embedded in $A_{\beta}$ via the obvious projection $\bigoplus_{\gamma \in \Gamma} A_{\gamma} \rightarrow A_{\beta}$. So let $E\left(V_{\gamma}\right)$ be an injective hull of $V_{\gamma}$ in $A_{\gamma}(\gamma \in \Gamma)$. By assumption, we can write $\Gamma=\Gamma_{1} \cup \Gamma_{2}$ for two disjoint infinite subsets $\Gamma_{i}$ of $\Gamma$. By the above argument, we can take, say, $\bigoplus_{\gamma \in \Gamma_{1}} A_{\gamma}$ to be injective. But then $\bigoplus_{\gamma \in \Gamma_{1}} E\left(S_{n_{\gamma}}\right) \cong \bigoplus_{\gamma \in \Gamma_{1}} E\left(V_{\gamma}\right)$ 
is also injective (since the latter is a direct summand of $\bigoplus_{\gamma \in \Gamma_{1}} A_{\gamma}$ ), contradicting ( $\sharp$ ) above. So we can write $T=A \oplus B$, where $A$ is extending and noninjective and $B$ is injective.

Now, by Zorn's lemma, choose a maximal subset $I$ of $\mathbb{N}$ such that $\left(\bigoplus_{n \in I} E\left(S_{n}\right)\right) \cap$ $B=0$. Then $\left(\bigoplus_{n \in I} E\left(S_{n}\right)\right) \oplus B$ is essential in $T$. Thus, $\bigoplus_{n \in I} E\left(S_{n}\right)$ embeds essentially in $A$ via the obvious projection from $A \oplus B$ onto $A$. So we may assume, without loss of generality, that $\bigoplus_{n \in I} E\left(S_{n}\right) \subseteq A$. If $I$ were finite, then we would have $A=\bigoplus_{n \in I} E\left(S_{n}\right)$, whence $A$ would be injective, contradicting the choice of $A$. So $A$ has infinite socle. Now let $E(A)$ be an injective hull of $A$ in $E(V)$. Then $E(A) \oplus B=E(V)$, so that $\frac{E(A)}{A}$ is colocal. Pick any element $x \in E(A)-A$ and an injective hull $K$ of $x R$ in $E(A)$. Then $K \cap A$ is a closed submodule of $A$ by Lemma 1. Since $A$ is extending, then $(K \cap A) \oplus A^{\prime}=A$ for some submodule $A^{\prime}$ of $A$. In the same way as argued above, $K \cap A$ contains a direct sum of injective hulls of simple modules essentially, and, again as above, since $K \cap A$ is not injective, it must have infinite socle. Since $x R$ is essential in $K$, this means $x R$ has infinite socle. Thus $R$ is not right q.f.d. This completes the proof.

The proof of Lemma 2 owes to a very useful observation of Beidar and Ke (see [1]). In the proof of the next result, we use a technique from a somewhat stronger version of the well-known Osofsky-Smith Theorem (see [11]), namely [3, 7.12]. Its adaptation here, however, is not straightforward.

Theorem 1. The following conditions are equivalent for a ring $R$ :

(i) $R$ satisfies the property $(*)_{r}$,

(ii) $R$ has finite type and right colocal type.

In this case $R$ is Artinian and right serial, and every right $R$-module is a direct sum of uniform modules.

Proof. $($ ii $) \Rightarrow(i)$. Since by finite type assumption, every right $R$-module is a direct sum of finitely generated indecomposable modules, each of which is now colocal (hence uniform), $(i)$ follows immediately.

$(i) \Rightarrow(i i)$. Let $R$ be a ring with property $(*)_{r}$. First we prove that $R$ is right q.f.d.

Assume the contrary. Then there exists a cyclic right $R$-module $H$ with infinite Goldie dimension. By assumption $(*)_{r}$, we may take $H$ to be extending. This implies that $H$ does not have a decomposition into indecomposable modules. Then we can easily obtain two sequences of modules $\left(X_{n}\right)_{n \in \mathbb{N}}$ and $\left(Y_{n}\right)_{n \in \mathbb{N}}$ such that for each $n \in \mathbb{N}$,

$$
X_{n} \neq 0, \bigoplus_{i=n+1}^{\infty} X_{i} \subseteq Y_{n}, \text { and } H=X_{1} \oplus \ldots \oplus X_{n} \oplus Y_{n}
$$

For each $n \in \mathbb{N}$, pick a maximal submodule $Z_{n}$ of $X_{n}$ and put $Z=\bigoplus_{n \in \mathbb{N}} Z_{n}$, $M=\frac{H}{Z}$, and $S_{n}=\frac{X_{n}+Z}{Z}$. Then $\left\{S_{n}: n \in \mathbb{N}\right\}$ is a local summand of $M$. By assumption, $M=A_{1} \oplus \ldots \oplus A_{t}$, where each $A_{i}$ is an extending module. Since simple modules have the exchange property, we have a decomposition

$$
M=S_{1} \oplus A_{(1,1)} \oplus \ldots \oplus A_{(t, 1)},
$$

where each $A_{(i, 1)}$ is a direct summand of $A_{i}$. Put $L_{1}=A_{(1,1)} \oplus \ldots \oplus A_{(t, 1)}$ (of course, only one of $A_{(i, 1)}$ is a proper submodule of $\left.A_{i}\right)$. Since $S_{1}$ is simple, there is an 
index $i_{1} \in\{1, \ldots, t\}$ and a direct summand $B_{1}$ of $A_{i_{1}}$ such that $B_{1} \oplus L_{1}=M$. Then $B_{1} \cong S_{1}$, so that $B_{1}$ is simple. Let $\pi: S_{1} \oplus L_{1} \rightarrow L_{1}$ be the obvious projection. Then $\left\{\pi\left(S_{n}\right): n=2,3, \ldots\right\}$ is a local summand of $L_{1}$. Thus, without loss of generality, we can assume that $\bigoplus_{n=2}^{\infty} S_{n} \subseteq L_{1}$. Similar to the above argument, we have a decomposition

$$
L_{1}=S_{2} \oplus A_{(1,2)} \oplus \ldots \oplus A_{(t, 2)},
$$

where each $A_{(i, 2)}$ is a direct summand of $A_{(i, 1)}$. Also, there exists some index $i_{2} \in\{1, \ldots, t\}$ and some direct summand $B_{2}$ of $A_{\left(i_{2}, 1\right)}$ with $B_{2} \oplus L_{2}=L_{1}$, where $L_{2}=A_{(1,2)} \oplus \ldots \oplus A_{(t, 2)}$. Thus $B_{2} \cong S_{2}$ so that $B_{2}$ is simple. Continuing in this manner we obtain a sequence $\left(i_{n}\right)_{n \in \mathbb{N}}$ of indices belonging to the set $\{1, \ldots, t\}$, sequences of modules $\left(L_{n}\right)_{n \in \mathbb{N}}$ and $\left(B_{n}\right)_{n \in \mathbb{N}}$, and descending chains of summands $\left(A_{(1, n)}\right)_{n \in \mathbb{N}}, \ldots,\left(A_{(t, n)}\right)_{n \in \mathbb{N}}$, such that for each $n \in \mathbb{N}, B_{n+1}$ is a direct summand of $A_{\left(i_{n+1}, n\right)}$, and

$$
L_{n}=A_{(1, n)} \oplus \ldots \oplus A_{(t, n)}=S_{n+1} \oplus L_{n+1}=B_{n+1} \oplus L_{n+1} .
$$

Now there exists some $t_{0} \in\{1, \ldots, t\}$ such that the set $\Gamma=\left\{k \in \mathbb{N}: i_{k}=t_{0}\right\}$ is infinite. Since $\left\{B_{n}: n \in \mathbb{N}\right\}$ is a local summand of $M,\left\{B_{n}: n \in \Gamma\right\}$ is a local summand of $A_{t_{0}}$. Taking an essential closure of $\bigoplus_{n \in \Gamma} B_{n}$ in $A_{t_{0}}$, we obtain a cyclic extending module $N$ such that $\operatorname{soc}(N)$ is infinitely generated and essential in $N$ and every finitely generated semisimple submodule of $N$ is a direct summand of $N$.

Now put $S=\operatorname{soc}(N)$. Then by the assumption $(*)_{r}, \frac{N}{S}$ has a nonzero extending summand, say $\frac{N^{\prime}}{S}$. Also $N^{\prime}=\bigoplus_{i \in I} K_{i}$ for some extending modules $K_{i}$. There exists some $i_{0} \in I$ such that $K_{i_{0}}$ is not semisimple. Let $K=K_{i_{0}}$. Also, since all finitely generated semisimple submodules of $K$ are direct summands of $N$, whence of $K, K$ has infinite socle. Since $\frac{K}{\operatorname{soc}(K)}$ is isomorphic to a direct summand of $\frac{N^{\prime}}{S}$, whence of $\frac{N}{S}, \frac{K}{\operatorname{soc}(K)}$ is cyclic as well as extending. Then, $K=G \oplus C$ for some semisimple submodule $G$ and cyclic submodule $C$. Note that $\operatorname{soc}(C)$ cannot be finitely generated. So $C$ is a cyclic extending module with infinite essential socle, and all finitely generated semisimple submodules of $C$ are direct summands of $C$, and $\frac{C}{\operatorname{soc}(C)}$ is also extending. We can write $\operatorname{soc}(C)=\bigoplus_{n \in \mathbb{N}} V_{n}$, where $V_{n}$ are some infinitely generated semisimple submodules of $C$. Since $C$ is extending, each $V_{n}$ is contained in a direct summand $D_{n}$ of $C$ essentially as well as properly (\#). Now let $D_{n}^{\prime}=\frac{D_{n}+\operatorname{soc}(C)}{\operatorname{soc}(C)}$. It is easy to see that $\sum_{n \in \mathbb{N}} D_{n}^{\prime}=\bigoplus_{n \in \mathbb{N}} D_{n}^{\prime}$. Since $\frac{C}{\operatorname{soc}(C)}$ is extending, there exists a direct summand $E^{\prime}$ of $\frac{C}{\operatorname{soc}(C)}$ containing $\bigoplus_{n \in \mathbb{N}} D_{n}^{\prime}$ essentially. Then there is a cyclic submodule $E$ of $C$ such that $E^{\prime}=\frac{E+\operatorname{soc}(C)}{\operatorname{soc}(C)}$.

We now claim that $E \cap D_{n} \neq 0$, for each $n \in \mathbb{N}$ : Assume to the contrary that $E \cap D_{n}=0$. We can write $E+\operatorname{soc}(C)=E \oplus P$ for some semisimple submodule $P$. Since $D_{n} \subseteq E+\operatorname{soc}(C)$, this means that $D_{n}$ can be embedded in $P$, a contradiction, since $D_{n}$ is not semisimple by $\left.{ }^{\sharp}\right)$ above. Thus $E \cap D_{n} \neq 0$. This implies that $E \cap \operatorname{soc}\left(D_{n}\right)=E \cap V_{n} \neq 0^{(\sharp \sharp)}$.

By assumption $(*)_{r}, E=E_{1} \oplus \ldots \oplus E_{s}$ for some cyclic extending modules $E_{i}$. For each $i \in \mathbb{N}$, let $\pi_{i}: \bigoplus_{n \in \mathbb{N}} V_{n} \rightarrow V_{i}$ be the natural projection. Note first that $\operatorname{supp}(\operatorname{soc}(E))=\left\{n \in \mathbb{N}: \pi_{n}(\operatorname{soc}(E)) \neq 0\right\}$ is infinite by ${ }^{(\sharp \sharp)}$ above. Hence, there exists some $k \in\{1, \ldots, s\}$ such that $\operatorname{supp}\left(\operatorname{soc}\left(E_{k}\right)\right)$ is infinite ${ }^{(\natural)}$. Now, if $U$ is a set of generators of $\operatorname{soc}\left(E_{k}\right)$, we have $\operatorname{supp}\left(\operatorname{soc}\left(E_{k}\right)\right)=\bigcup_{x \in U} \operatorname{supp}(x)$. So, if $U$ were finite, $\operatorname{supp}\left(\operatorname{soc}\left(E_{k}\right)\right)$, too, would be finite. Therefore $\operatorname{soc}\left(E_{k}\right)$ is infinitely generated. Then let $\operatorname{soc}\left(E_{k}\right)=\bigoplus_{\alpha \in \Lambda} L_{\alpha}$, where $|\Lambda|=\infty$ and $L_{\alpha}$ are simple modules. By $\left.{ }^{(}\right)^{\text {, }}$ 
and since each $\operatorname{supp}\left(L_{\alpha}\right)$ is finite, we can inductively select indices $\alpha_{n}(n \in \mathbb{N})$ such that, for each $n \in \mathbb{N}$,

$$
\max \left(\operatorname{supp}\left(L_{\alpha_{n+1}}\right)\right)>\max \left(\operatorname{supp}\left(L_{\alpha_{n}}\right)\right) .
$$

This implies, in particular, that for each $n \in \mathbb{N}$,

$$
\max \left(\operatorname{supp}\left(L_{\alpha_{n+1}}\right)\right)>n .
$$

Now since $E_{k}$ is an extending module, we can find some direct summand $L$ of $E_{k}$ containing $\bigoplus_{n \in \mathbb{N}} L_{\alpha_{n}}$ essentially. This containment is proper since $L$ is cyclic. Let $L^{\prime}=\frac{L+\operatorname{soc}(C)}{\operatorname{soc}(C)}$. So, $L^{\prime} \neq 0$ obviously, whence

$$
L^{\prime} \cap\left(\bigoplus_{n \in \mathbb{N}} D_{n}^{\prime}\right) \neq 0
$$

Now let $n \in \mathbb{N}$.

Claim: $L \cap\left(\bigoplus_{i=1}^{n} V_{i}\right) \subseteq \bigoplus_{i=1}^{n+1} L_{\alpha_{i}}$.

Proof of claim: Assume the contrary and let $x \in L \cap\left(\bigoplus_{i=1}^{n} V_{i}\right)$ be with $x=$ $l_{\alpha_{1}}+\ldots+l_{\alpha_{m}}$, where $l_{\alpha_{i}} \in L_{\alpha_{i}}, l_{\alpha_{m}} \neq 0$ and $m>n+1$.

Since $L_{\alpha_{m}}$ is simple and $l_{\alpha_{m}} \neq 0, l_{\alpha_{m}}$ generates $L_{\alpha_{m}}$. Put $w^{\prime}=\max \left(\operatorname{supp}\left(L_{\alpha_{m}}\right)\right)$ and $w=\max \left(\operatorname{supp}\left(L_{\alpha_{m-1}}\right)\right)$. Note that $w^{\prime}=\max \left(\operatorname{supp}\left(l_{\alpha_{m}}\right)\right)$ too. Then, by choice of $\alpha_{n}$ and ${ }^{(\dagger)}$, we have

$$
w^{\prime}>w=\max (\operatorname{supp}(Q)),
$$

where $Q=\left(\bigoplus_{i=1}^{n} V_{i}\right)+\left(\bigoplus_{j=1}^{m-1} L_{\alpha_{j}}\right)$. But since $l_{\alpha_{m}} \in Q$, we have a contradiction. This proves our claim.

Consequently, $\operatorname{soc}\left(L \cap\left(\bigoplus_{i=1}^{n} D_{i}\right)\right)=L \cap\left(\bigoplus_{i=1}^{n} V_{i}\right)$ is finitely generated, whence a direct summand of $L$, and thus of $L \cap\left(\bigoplus_{i=1}^{n} D_{i}\right)$. However, since $\operatorname{soc}\left(L \cap\left(\bigoplus_{i=1}^{n} D_{i}\right)\right)$ is essential in $L \cap\left(\bigoplus_{i=1}^{n} D_{i}\right)$, this means that

$$
L \cap\left(\bigoplus_{i=1}^{n} D_{i}\right) \subseteq \operatorname{soc}(C) .
$$

Hence, $L \cap\left(\bigoplus_{i=1}^{\infty} D_{i}\right) \subseteq \operatorname{soc}(C)$. This contradicts ${ }^{(\ddagger)}$ above. Therefore $R$ must be right q.f.d.

By Lemma 2 and the above argument, we now have that $R$ is a right Noetherian ring. Since every extending module over a right Noetherian ring has a decomposition into indecomposable extending (i.e., uniform) modules by [9], every right $R$-module now has such a decomposition by the assumption $(*)_{r}$. Thus $R$ is right pure-semisimple, so that every right $R$-module is a direct sum of finitely generated uniform modules by [19]; whence $R$ is right Artinian and indecomposable right $R$-modules are colocal. Then, up to isomorphism, there are finitely many simple right $R$-modules, and thus, finitely many indecomposable injective right $R$-modules. Since each uniform right $R$-module can be embedded in one of those indecomposable injective modules, $R$ then has (right) bounded type and then, by [18] (also see $[12]$ ), finite type. This completes the proof of $(i) \Rightarrow(i i)$.

Now, whenever $R$ satisfies one of the above equivalent conditions, we have $R_{R}=\bigoplus_{i=1}^{n} e_{i} R$ for some local idempotents $e_{i}$. Every factor of each $e_{i} R$ is then indecomposable, whence uniform. This implies that $e_{i} R$ are uniserial as right $R$ modules. Therefore $R$ is right serial. The proof is now complete. 
In [6], Fuller investigates rings of finite type and with all left indecomposable modules quasi-injective, namely rings of left invariant module type. Artinian serial rings, rings of right invariant module type, and finite dimensional algebras (over a field) of left local type (i.e., every indecomposable left module is local) are among examples of rings with $(*)_{r}$ (see [14, [15], [16, and [17).

Corollary 1. A ring $R$ is of right invariant module type if and only if every right $R$-module is a direct sum of quasi-injective modules.

The property $(*)_{r}$ does not imply $(*)_{l}$, as the following example due to Singh shows:

Example 1 ([13, Example 1]). Let $F \subseteq K$ be an extension of finite dimensional division rings, with $[K: F]=2$. Let $R=\left(\begin{array}{cc}F & K \\ 0 & K\end{array}\right)$. Then $R$ is an Artinian right serial ring satisfying $(*)_{r}$ by $\left[13\right.$, Theorem 3.6]. Thus, $R$ does not satisfy $(*)_{l}$ by Theorem 1 .

The above example also shows that rings with $(*)_{r}$ are not necessarily left serial. Now we are ready to give our next result:

Corollary 2. The following conditions are equivalent for a ring $R$ :

(i) $R$ is Artinian serial,

(ii) $R$ has property $(*)$,

(iii) $R$ is left (resp. right) serial with property $(*)_{r}$ (resp. $\left.(*)_{l}\right)$,

(iv) $R$ has $(*)_{r}$ and every 2 -generated uniform right $R$-module is uniserial,

(v) $R$ is right or left $Q F-3$ with property $(*)_{r}$.

Proof. The equivalence of $(i),(i i)$, and (iii) follows from Theorem 1

Now assume $(i v)$. Then $R$ is Artinian by assumption and Theorem 1, and every finitely generated uniform right $R$-module is uniserial. Then finitely generated right $R$-modules are serial by the $(*)_{r}$ assumption. Now $(i)$ follows by [7, Theorem 25.4.2. $\left.\left(1_{b i s}\right)\right]$.

Finally, the equivalence of $(i)$ and $(v)$ follows from Theorem 1 and [10, Theorem 4.4].

\section{ACKNOWLEDGEMENTS}

We would like to thank Professor N. V. Dung for kindly pointing out the argument regarding bounded type at the end of the proof of Theorem 1 We also thank the referee for a very careful reading of the manuscript and helpful comments.

\section{REFERENCES}

[1] K. I. Beidar and W.-F. Ke, On essential extensions of direct sums of injective modules, Arch. Math. (Basel), 78 (2002), No. 2, 120-123. MR1888412 (2003a:16006)

[2] N. V. Dung and P. F. Smith, Rings for which certain modules are CS, J. Pure Appl. Algebra, 102 (1995), No. 3, 273-287. MR1354992 (96i:16010)

[3] N. V. Dung, D. V. Huynh, P. F. Smith and R. Wisbauer, Extending Modules, Pitman Research Notes in Mathematics Series, 313, Longman Sci. \& Tech., Harlow; copublished in the U.S. with Wiley, New York, 1994. MR1312366 (96f:16008)

[4] D. V. Huynh and S. T. Rizvi, Characterizing rings by a direct decomposition property of their modules, J. Aust. Math. Soc., 80 (2006), No. 3, 359-366. MR.2236043 (2007i:16010)

[5] D. V. Huynh and B. J. Müller, Rings over which direct sums of $C S$ modules are CS, Advances in Ring Theory, Eds. S. K. Jain and S. T. Rizvi, Birkhäuser Boston, Boston, MA, 1997, 151159. MR,1602657 (98i:16008) 
[6] K. R. Fuller, Rings of left invariant module type, Comm. Algebra, 6 (1978), No. 2, 153-167. MR0472908 (57:12593)

[7] C. Faith, Algebra II, Ring Theory, Grundlehren der Mathematischen Wissenschaften, Vol. 191, Springer, Berlin-New York, 1976. MR0427349 (55:383)

[8] R. Makino, Balanced modules and rings of left colocal type, Tsukuba J. Math., 15 (1991), No. 2, 465-477. MR.1138199 (93b:16028)

[9] M. Okado, On the decomposition of extending modules, Math. Japonica, 29 (1984), 939-941. MR803451 (86k:16013)

[10] K. Oshiro, Theories of Harada in Artinian rings and applications to classical Artinian rings, International Symposium on Ring Theory, Eds. G.F. Birkenmeier, J.K. Park and Y.S. Park, Birkhäuser Boston, Boston, MA, 2001, 279-301. MR1851210 (2002g:16031)

[11] B. L. Osofsky and P. F. Smith, Cyclic modules whose quotients have all complement submodules direct summands, J. Algebra, 139 (1991), No. 2, 342-354. MR.1113780 (92f:16030)

[12] M. Prest, Duality and pure-semisimple rings, J. London Math. Soc. (2), 38 (1988), No. 3, 403-409. MR.972125 (89m:16059)

[13] S. Singh, Indecomposable modules over Artinian right serial rings, Advances in Ring Theory, Eds. S. K. Jain and S. T. Rizvi, Birkhäuser Boston, Boston, MA, 1997, 295-304. MR.1602697 (98m:16023)

[14] S. Sumioka, On Artinian rings of right local type, Math. J. Okayama Univ., 29 (1987), 127146. MR 936736 (89d:16021)

[15] S. Sumioka, Tachikawa's theorem on algebras of left colocal type, Osaka J. Math., 21 (1984), 629-648. MR759486 (86a:16028)

[16] H. Tachikawa, On rings for which every indecomposable right module has a unique maximal submodule, Math. Z., 71 (1959), 200-222. MR0107662 (21:6385)

[17] H. Tachikawa, On algebras of which every indecomposable representation has an irreducible one as the top or the bottom Loewy constituent, Math. Z., 75 (1960/61), 215-227. MR0124356 (23:A1670)

[18] B. Huisgen-Zimmermann and W. Zimmermann, On the sparsity of representations of rings of pure global dimension zero, Trans. Amer. Math. Soc., 320 (1990), No. 2, 695-711. MR965304 (90k:16036)

[19] B. Huisgen-Zimmermann, Rings whose right modules are direct sums of indecomposable modules, Proc. Amer. Math. Soc., 77 (1979), No. 2, 191-197. MR542083 (80k:16041)

Department of Mathematics, University of Rio Grande, Rio Grande, Ohio 45674

E-mail address: noyaner@yahoo.com 